\section{Antipsychotika in der Schwangerschaft können dem Nachwuchs schaden}

Nicht immer können psychisch kranke Frauen in der Schwangerschaft auf Medikamente verzichten. Gerade bei Antipsychotika sollten sie aber vorsichtig sein: Eine Studie liefert Hinweise auf motorische Defizite beim Nachwuchs nach Antipsychotika-Einnahme in der Schwangerschaft.

A ntipsychotika werden bekanntlich längst nicht mehr nur bei Psychosen verabreicht, sondern auch bei vielen anderen psychischen Störungen wie Depressionen, Bipolar-Erkrankungen oder Angststörungen. Die Zahl der Verschreibungen hat sich in den USA in den vergangenen zehn Jahren entsprechend fast verdreifacht, dort zählen diese Arzneien inzwischen zu den am häufigsten verordneten Substanzen. Allerdings, so berichten US-Forscher um Katrina Johnson von der Universität in Atlanta, gebe es bislang kaum Daten zur Sicherheit in der Schwangerschaft. Diesen Mangel wollte das Team um die Psychiaterin mit einer prospektiven Studie zumindest ein Stück weit beheben. Die Forscher begleiteten über 300 Schwangere und untersuchten deren Kinder sechs Monate nach der Geburt.

Die Frauen hatten alle eine psychische Erkrankung, die Mütter von 202 Kindern nahmen während der Schwangerschaft Antidepressiva, die von 22 Kindern Antipsychotika, die Mütter der übrigen 85 Kinder verzichteten in der Schwangerschaft auf psychotrope Arzneimittel. Untersucht wurden die Babys mit einem speziellen neuromotorischen Test, der Infant Neurological International Battery (INFANIB). Dabei wird auf Tonus, Reflexe, Körperhaltung und Motorik geachtet. Maximal möglich sind 100 Punkte, Werte über 72 Punkte gelten für Babys mit sechs Monaten als normal, Werte unter 55 Punkte als pathologisch, dazwischen liegt ein Übergangsbereich.

\section{Nur $19 \%$ mit normaler Motorik}

Im Schnitt erreichten die Kinder ohne Psychopharmaka-Kontakt 71 und die mit Antidepressiva-Exposition 69 Punkte. Am niedrigsten war der Wert mit 65 Punkten jedoch bei Kindern, deren Mütter in der Schwangerschaft Antipsychoti- ka nahmen - die Unterschiede zu den anderen Gruppen waren signifikant. Von den Kindern mit Antipsychotika-Kontakt hatten nur $19 \%$ normale Werte im Test, $32 \%$ waren es bei denjenigen mit Antidepressiva-Exposition und $50 \%$ bei den Kindern ohne Psychopharmaka im Mutterleib. Doch nicht nur Antipsychotika, sondern auch die Schwere der Erkrankung sowie vorangegangene depressive und psychotische Störungen der Mutter erwiesen sich als Risikofaktor für eine schlechte neuromotorische Funktion des Nachwuchses.

Insgesamt, so die Forscher, scheint eine psychische Erkrankung der Mutter per se ungünstig für die prä- und perinatale Entwicklung der Kinder zu sein, Antipsychotika können diesen Effekt offenbar noch verstärken. Wie viel die Erkrankung und wie viel die Medikation zur ungünstigen Entwicklung beiträgt, lässt sich allerdings nicht klar abgrenzen. Da Neuroleptika Dopaminrezeptoren blockieren, erscheint ein Einfluss auf die Motorik des werdenden Kindes zumindest plausibel.

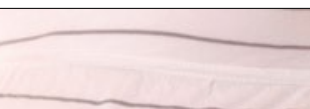

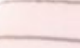

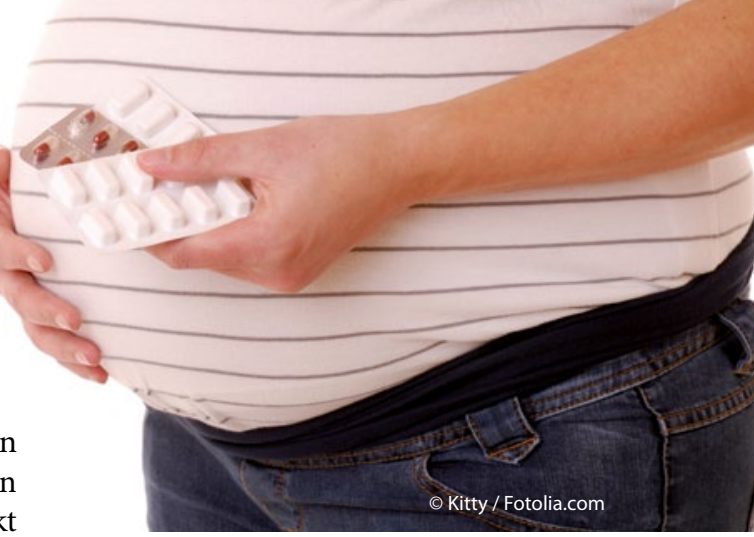

Nehmen Frauen in der Schwangerschaft Antipsychotika, müssen sie mit motorischen Defiziten beim Nachwuchs rechnen.

\section{Antipsychotika am häufigsten gegen Angststörungen}

Mit Blick auf die Daten raten die Forscher dazu, zusammen mit schwangeren Frauen noch gründlicher als bisher zu diskutieren, ob und welche Psychopharmaka in der Schwangerschaft wirklich nötig sind. Ein interessanter Nebenaspekt: Nur zwei der Mütter nahmen die Antipsychotika aufgrund einer Psychose, die übrigen gegen andere psychische Erkrankungen, am häufigsten gegen Angststörungen. Neun erhielten den Klassiker Haloperidol, 13 bekamen atypische Neuroleptika, eine Schwangere nahm beides.

Thomas Müller

Johnson C et al. Arch Gen Psychiatry, Apr

2012; doi:10.1001/archgenpsychiary.2012.160

\title{
Schützen Statine auch vor Parkinson-Erkrankung?
}

\begin{abstract}
Ursprünglich erfunden zur Cholesterinsenkung und Prävention von kardiovaskulären Erkrankungen, werden Statine inzwischen noch ganz andere Wirkungen nachgesagt. Eine neue Studie bringt diese Lipidsenker jetzt sogar mit der Verhinderung von Parkinson-Erkrankungen in Verbindung.
\end{abstract}

tatine zählen zu den weltweit am häufigsten verordneten Medikamenten. Sie hemmen bekanntlich die HMG-Coenzym-A-Reduktase, ein Schlüsselenzym in der Cholesterinsynthese. Viel ist über mögliche „pleiotrope“, etwa antiinflammatorische und immunmodulierende Wirkeigenschaften dieser potenten Lipidsenker diskutiert worden. Daraus ist die
Hypothese erwachsen, dass Statine auch als neuroprotektive Wirkstoffe von Nutzen sein könnten, so etwa zur Vorbeugung einer Parkinson-Erkrankung. Die bisherigen Studien - zumeist Analysen von Registerdaten - ergeben dazu allerdings ein sehr gemischtes Bild. Ein Forscherteam um Xiang Gao von der HarvardUniversität in Boston hat sich deshalb bei 